\title{
Attenuated mRNA expression of inflammatory mediators in neonatal rat lung following lipopolysaccharide treatment
}

This article was published in the following Dove Press journal:

Journal of Inflammation Research

6 September 2012

Number of times this article has been viewed

\section{Valerie Le Rouzic \\ Kari Wiedinger \\ Heping Zhou}

Department of Biological Sciences, Seton Hall University, South Orange, NJ, USA
Correspondence: Heping Zhou Department of Biological Sciences, Seton Hall University, 400 South Orange Avenue, South Orange, NJ 07079, USA Tel +l 9732752889

Fax + I 9732752905

Email heping.zhou@shu.edu
Abstract: Neonates are known to exhibit increased susceptibility to bacterial and viral infections and increasing evidence demonstrates that the increased susceptibility is related to their attenuated immune response to infections. The lung is equipped with an innate defense system involving both cellular and humoral mediators. The present study was performed to characterize the expression of inflammatory mediators in the lung of neonatal rats in comparison with older animals. Rats at postnatal day 1 (P1), P21, and P70 were treated with saline or $0.25 \mathrm{mg} / \mathrm{kg}$ lipopolysaccharide (LPS) via intraperitoneal injection. Two hours later, animals were sacrificed and the transcriptional response of key inflammatory mediators and enzyme activity of myeloperoxidase (MPO) in the lung of these animals were examined. LPS-induced messenger RNA (mRNA) expression of pro-inflammatory cytokines, namely interleukin (IL)-1 $\beta$, IL-6, and tumor necrosis factor- $\alpha$, antiinflammatory cytokines, namely IL-10 and IL-1 receptor antagonist (IL-1 ra), and chemokines, namely macrophage inflammatory protein (MIP)-1 $\beta$, MIP-2, and monocyte chemotactic protein-1, in P1 lung was much reduced compared to that in P21 and P70 animals at 2 hours postinjection. These data suggest that LPS-induced transcriptional response of cytokines and chemokines was much reduced in P1 lung even though the protein levels of these genes were not ascertained and mRNA levels of these genes may not reflect their final protein levels. MPO activity in LPS-treated P1 lung was also significantly attenuated compared to that in LPS-treated P70 lung, suggesting impaired neutrophil infiltration in P1 lung at 2 hours following LPS treatment. In parallel, the baseline mRNA expression of LPS-binding protein (LBP) in P1 lung was much lower than that in P21 and P70 lungs. While the protein level of LBP was not examined and the mRNA level of LBP may not reflect its final protein level, the reduced transcriptional response of cytokines and chemokines in P1 lung at 2 hours following LPS treatment may be attributed to lower LBP expression in P1 lung as compared to P21 and P70 lungs.

Keywords: innate immunity, lung, LPS, development, inflammatory mediators

\section{Introduction}

To preserve homeostasis and protect itself from injury, the lung is equipped with an innate defense system involving both cellular and humoral mediators. Both leukocytes and epithelial cells present in the lung express an array of pattern recognition receptors such as toll-like receptors (TLRs) and antimicrobial compounds. Upon binding to appropriate pathogen-associated molecular patterns (PAMPs), TLRs transmit signals to the nucleus of these cells and initiate an immune response. For example, TLR-2 associates with TLR-1 or TLR-6 to recognize triacyl- and diacyl-lipopeptides, respectively, TLR-4 recognizes lipopolysaccharides (LPS), and TLR-5 recognizes flagellin (Figure 1). ${ }^{1}$ 


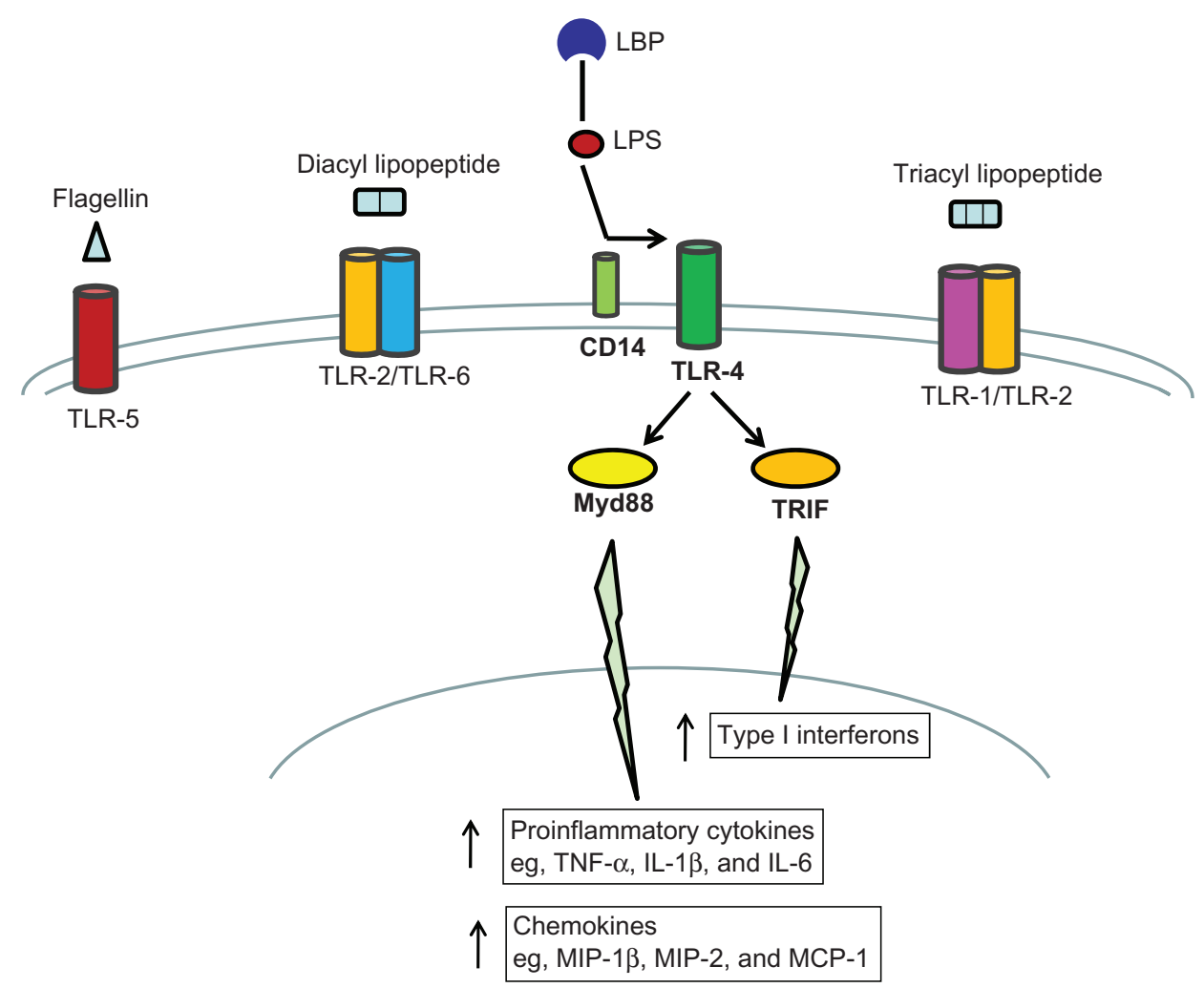

Figure I Overview of LPS/TLR-4 signaling pathway.

Notes: LPS is delivered to CDI4 by LBP, which in turn transfers LPS to TLR-4. Upon LPS binding, TLR-4 initiates the MyD88-dependent and MyD88-independent pathways. The MyD88-dependent pathway ultimately upregulates the production of inflammatory mediators including cytokines and chemokines, and the MyD88-independent pathway ultimately increases the production of Type I interferons.

Abbreviations: LBP, LPS-binding protein; LPS, lipopolysaccharide; MCP, monocyte chemotactic protein; MIP, macrophage inflammatory protein; MyD88, myeloid differentiation marker 88; TLR, toll-like receptor; TNF- $\alpha$, tumor necrosis factor- $\alpha$; TRIF, TIR domain-containing adaptor inducing IFN- $\beta$.

LPS, an endotoxin from the outer membrane of Gramnegative bacteria, first binds to LPS-binding protein (LBP), which facilitates the binding of LPS to membrane CD14 (mCD14). ${ }^{2} \mathrm{mCD} 14$ then transfers LPS to TLR-4, which activates both myeloid differentiation markers (MyD) 88-dependent and MyD88-independent intracellular signaling cascades. The MyD88-dependent pathway ultimately upregulates the production of inflammatory mediators including cytokines and chemokines, and the MyD88-independent pathway ultimately leads to increased production of type I interferons (Figure 1). ${ }^{3}$ The proinflammatory cytokines, such as tumor necrosis factor (TNF)- $\alpha$, interleukin (IL)- $1 \beta$, and IL-6, are primarily responsible for initiating an effective defense against exogenous pathogens while anti-inflammatory cytokines, such as IL-10 and IL-1 receptor antagonist (IL-1ra), which is a naturally occurring antagonist of IL-1, are crucial for downregulating the inflammatory process. ${ }^{4}$ Chemokines are directly involved in the recruitment of immune cells, such as neutrophils and T cells, to the site of infection. ${ }^{5}$

Neonatal sepsis is one of the major health problems throughout the world, with an estimated 30 million newborns acquiring infections every year. ${ }^{6}$ In the United States, bacterial sepsis affects up to 32,000 live births annually. ${ }^{7}$ There is evidence that increased neonatal susceptibility to bacterial and viral infections may be related to their attenuated immune response. Studies have found that cord blood monocytes are less responsive to bacterial lipopeptides, double-stranded RNA, and LPS that act through TLR-2, TLR-3, and TLR-4, respectively, than adult peripheral blood monocytes (PBMCs). ${ }^{8-10}$ Cord blood dendrocytes have also been shown to express lower levels of the gamma-chain of the IL-2 receptor (CD132) and CD86 co-stimulatory molecules, suggesting their relative immaturity as compared to adult peripheral blood dendrocytes. ${ }^{11}$

It has been reported that TLR-4 expression and cytokine responsiveness of fetal lung to LPS become detectable at embryonic day 17 and further increase after birth in mice. ${ }^{12}$ Pulmonary neutrophil recruitment following bacterial infections has also been reported to be impaired in neonatal rats compared to adult rats. ${ }^{13,14}$ Additionally, IL-10 expression is only minimally augmented in neonatal alveolar macrophages 
compared with those from adult rats. ${ }^{15}$ These studies suggest that the pulmonary innate immunity in the neonates may be relatively immature.

The present study was undertaken to compare the induction of inflammatory mediators in the lung of animals at different stages of postnatal development following LPS treatment in order to understand the mechanisms underlying increased susceptibility of neonates to infections. We treated animals at postnatal day 1 (P1), P21, and P70 with $0.25 \mathrm{mg} / \mathrm{kg}$ LPS and examined the expression of various inflammatory mediators in the lung of these animals. Animals were treated with $0.25 \mathrm{mg} / \mathrm{kg}$ LPS for 2 hours based on our previous finding that $0.25 \mathrm{mg} / \mathrm{kg}$ LPS was able to induce an inflammatory response in rat liver at P1, P21, and P70 at 2 hours postinjection. ${ }^{16}$

\section{Materials and methods}

\section{Animals}

Young adult male and female Sprague-Dawley ${ }^{\circledR}$ rats (Harlan Inc, Indianapolis, IN) were maintained in a temperature- and humidity-controlled facility on a $12 \mathrm{~h}$ day/night cycle and fed a standard rat diet with water ad libitum. The female rats were bred with male rats, and the ones with vaginal plugs were moved to separate cages. Animals were treated with either saline or $0.25 \mathrm{mg} / \mathrm{kg}$ LPS (Salmonella typhimurium; Sigma Aldrich, St Louis, MO) via intraperitoneal (ip) injection (four animals per group) at P1, P21 (the day of weaning), or P70 (young adult). ${ }^{17}$ At the end of treatment, the animals were sacrificed and their lung tissues were dissected. Animal procedures were approved by the Institutional Animal Care and Use Committee (IACUC) at Seton Hall University.

\section{Total RNA extraction}

Total RNA was isolated from the dissected lung tissues using the TRIzol reagent (Invitrogen, Grand Island, NY) according to the manufacturer's instructions, dissolved in RNase-free water, and stored at $-80^{\circ} \mathrm{C}$.

\section{cDNA synthesis by reverse transcription (RT)}

Total RNA was used in RT reactions with ImProm-II ${ }^{\mathrm{TM}}$ reverse transcriptase (Promega, Madison, WI) and oligo $(\mathrm{dT})^{12-18}$ (Affymetrix, Cleveland, $\mathrm{OH}$ ) primers. All samples were set up from the same RT master mix and reverse transcribed under the same conditions at $42^{\circ} \mathrm{C}$ for 60 minutes to minimize differences in RT efficiency. ${ }^{16}$

\section{Quantitation by real-time polymerase chain reaction (PCR)}

To measure the relative mRNA levels of TLR-2, TLR-4, TLR-5, TLR-6, and LBP, real-time PCR was performed in $20 \mu \mathrm{L}$ reactions with 1 X SYBR Green master mix (Applied Biosystems, Foster City, CA) and $1 \mu \mathrm{L}$ of cDNA diluted 1:2 as PCR template together with $500 \mu \mathrm{M}$ appropriate specific sense and anti sense primers. $\beta$-actin, a housekeeping gene, was used as the control. The sense and antisense primers for rat TLR-2 were 5'-GGA-AGC-AGG-TGACAA-CCA-TT-3' and 5'-CGC-CTA-AGA-GCA-GGATCA-AC-3', respectively; the sense and antisense primers for rat TLR-4 were 5'-TGC-TCA-GAC-ATG-GCA-GTT-TC-3' and 5'-TCA-AGG-CTT-TTC-CAT-CCA-AC-3', respectively; the sense and antisense primers for rat TLR-5 were $5^{\prime}$ - TCTTCG-AAC-TTC-GGC-TGT-TT-3' and 5'-TGT-GAA-TCTCGT-TGG-CAG-AG-3', respectively; the sense and antisense primers for rat TLR-6 were 5'-AAG-ATG-ACA-TTC-GGGTTT-GC-3' ${ }^{\prime}$ and 5'-TCR-GGA-TGA-AGT-GG-GAG-AC-3', respectively; the sense and antisense primers for rat $\mathrm{LBP}$ were 5'-CCG-GGT-TTA-CCA-CCA-GGC-CG-3', and 5'-TTC-GTG-ACC-ACG-CCA-AGC-CG-3', respectively; and the sense and antisense primers for rat $\beta$-actin were 5'-AGC-CAT-GTA-CGT-AGC-CAT-CC-3' and 5'-AGC-CATGTA-CGT-AGC-CAT-CC-3', respectively. The PCR reactions were denatured at $95^{\circ} \mathrm{C}$ for 10 minutes with 40 cycles of amplification and quantification followed by a melting curve program according to the manufacturer's instructions in a StepOnePlus ${ }^{\mathrm{TM}}$ thermal cycler (Applied Biosystems). The generation of only the correct size amplification products was confirmed by agarose gel electrophoresis.

Real-time PCR data were analyzed by the ABI StepOne software supplied by Applied Biosystems. Ct-values of all samples were normalized to the $\mathrm{Ct}$ values of $\beta$-actin $(\Delta \mathrm{Ct})$ and $\Delta \mathrm{Ct}$ values of one of the saline control samples ('baseline' expression) were subtracted $(\Delta \Delta \mathrm{Ct})$. The expression of the control sample used to calculate $\Delta \Delta \mathrm{Ct}$ was set to 1 and values of other samples were plotted as fold expression of the baseline $2^{-\Delta \Delta \mathrm{Ct}}$.

\section{Quantitation by semi-quantitative PCR}

To measure the relative mRNA levels of CD14, MyD88, TNF- $\alpha$, IL-1 $\beta$, IL-6, IL-10, IL-1 ra, macrophage inflammatory protein (MIP)-1 $\beta$, MIP-2, and monocyte chemotactic protein (MCP)-1, cDNA samples were used to conduct PCR amplifications using appropriate specific sense and antisense primers. ${ }^{17,18}$ The sense and antisense primers for rat MyD88 were 5'-CCC-CTG-ACA-TGC- 
CTA-TCA-CT-3' and 5'-TGT-CCC-AAA-GGA-AACACA-CA- $3^{\prime}$, respectively; the sense and antisense primers for rat IL-10 were 5'-GGG-AAG-CAA-CTG-AAA-CTTCG-3' and 5'-GCT-TTC-GAG-ACT-GGA-AGT-GG-3', respectively; the sense and antisense primers for rat IL-1 ra

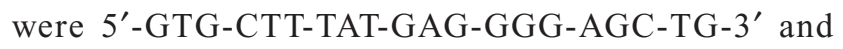
5'-GAG-CCA-TGT-TTG-CAC-AGA-GA-3', respectively; and the sense and antisense primers for rat MCP-1 were 5'-ATG-ATC-CCA-ATG-AGT-CGG-3' and 5'-ACAGAA-GTG-CTT-GAG-GTG-3', respectively. The PCR reactions were carried out by heating the samples to $94^{\circ} \mathrm{C}$ for 5 minutes, followed by appropriate number of cycles of denaturation at $94^{\circ} \mathrm{C}$ for 30 seconds, annealing at $57^{\circ} \mathrm{C}$ for 30 seconds, and extension at $72^{\circ} \mathrm{C}$ for 30 seconds. After the final cycle, the PCR reactions were extended for another 7 minutes. The optimum number of cycles was 25 cycles for MyD88 and CD14, 26 cycles for TNF- $\alpha$ and MIP-1 $\beta, 27$ cycles for IL-1 $\beta, 28$ cycles for IL- 6 and MCP-1, 29 cycles for MIP-2, 32 cycles for IL-10 and IL-1ra, and 21 cycles for $\beta$-actin. After running PCR products on a $2.0 \%$ agarose gel, the gel image was recorded using a UVP GelDoc-It ${ }^{\mathrm{TM}}$ imaging system (UVP, Upland, CA) and digitized using VisionWorks ${ }^{\mathrm{TM}}$ LS software (UVP). The relative intensities for genes of interest were normalized to the intensity of $\beta$-actin in the same sample. ${ }^{16,18}$

\section{Myeloperoxidase (MPO) activity assay}

Fifty micrograms of lung tissue samples from LPS- and saline-treated animals were homogenized in $300 \mu \mathrm{L}$ of $5 \mathrm{mM}$ potassium phosphate-buffered saline $(\mathrm{pH}=6.0)$ followed by centrifugation at $14,000 \mathrm{rpm}$ for 15 minutes at $4^{\circ} \mathrm{C}$. The pellets were then extracted in $300 \mu \mathrm{L}$ of $50 \mathrm{mM}$ potassium phosphate-buffered saline $(\mathrm{pH}=6.0)$ with $0.5 \%$ hexadecyltrimethylammonium bromide followed by 20 seconds of sonication and three cycles of freeze/thaw. The samples were then centrifuged at $14,000 \mathrm{rpm}$ for 15 minutes at $4{ }^{\circ} \mathrm{C} .{ }^{19}$ Protein concentrations of the supernatants were determined using bicinchoninic acid (BCA) protein assay reagent from Pierce Biotechnology Inc (Rockford, IL) ${ }^{17}$ MPO activity was then determined by adding $10 \mu \mathrm{L}$ of supernatant or equal volume of MPO standard solutions $(0,5,10,20$, and $40 \mathrm{U} /$ $\mathrm{mL})$ to $990 \mu \mathrm{L}$ of $50 \mathrm{mM}$ potassium phosphate-buffered saline $(\mathrm{pH}=6.0)$ containing $0.167 \mathrm{mg} / \mathrm{mL}$ of $o$-dianisidine and $0.0005 \%$ of $\mathrm{H}_{2} \mathrm{O}_{2}$ and recording the absorbance at $460 \mathrm{~nm}$ at 2 minutes after initiation of the reaction. ${ }^{20} \mathrm{MPO}$ activity of the lung tissue samples was determined from the standard curve and expressed as unit of MPO activity per mg of protein in the lung tissue extract $(\mathrm{U} / \mathrm{mg})$.

\section{Statistical analysis}

All data were analyzed using two-way analysis of variance (ANOVA) followed by Bonferroni's post-test (GraphPad Prism, La Jolla, CA). Results of $P<0.05$ were considered statistically significant.

\section{Results \\ Expression of TLRs in the lung of postnatal animals}

To investigate how the pulmonary innate immunity develops during the postnatal period, animals at P1, P21, and P70 were treated with saline or $0.25 \mathrm{mg} / \mathrm{kg}$ LPS via intraperitoneal (ip) injection. Two hours after the injection, the animals were sacrificed, their lung tissues were dissected, and total RNA was extracted from the lung tissues for determination of expression levels of various immune mediators. The animals stimulated with saline were used as negative controls.

We first examined the relative mRNA levels of TLR-2, TLR-4, TLR-5, and TLR-6 using real-time reverse transcription PCR. While the baseline mRNA levels of TLR-2 and TLR-5 were comparable in the lung of P1, P21, and P70 animals (Figure 2A and C), TLR-4 and TLR-6 in P1 lung was 3.93- and 2.71-fold lower than that in P70 lung, respectively (Figure 2B and D and Table 1). LPS treatment did not significantly alter the mRNA expression of TLR-2, TLR-4, TLR-5, and TLR-6 in P1 lung, but increased the mRNA level of TLR-2 by 6.18-fold in P21 lung without significant effects on the mRNA levels of TLR-4, TLR-5, and TLR-6. In contrast, LPS treatment significantly augmented the mRNA expression of TLR-2 by 8.59 -fold, TLR- 5 by 1.81 -fold, and TLR- 6 by 2.56 -fold, and attenuated the mRNA expression of TLR-4 by 1.75-fold in P70 lung at 2 hours post-injection (Table 1). The relative mRNA levels of TLR-2, TLR-4, TLR-5, and TLR-6 in LPS-treated P70 lung were 16.82-, 2.44-, 2.26-, and 6.88-fold higher than that in LPS-treated P1 lungs, respectively (Figure 2 and Table 1).

\section{Expression of upstream TLR-4 signaling mediators in the lung of postnatal animals}

We then examined the mRNA expression of key upstream mediators of the TLR-4 signaling pathway. The relative mRNA levels of CD14 and MyD88 were determined using semi-quantitative reverse transcription PCR and the relative mRNA level of LBP was determined using real-time reverse transcription PCR. The baseline mRNA levels of CD14 and MyD88 were comparable in P1, P21, and P70 lungs, yet the baseline mRNA level of LBP in P1 lung was 5.19 and 5.99-fold lower than that in P21 and P70 lung, respectively 
A

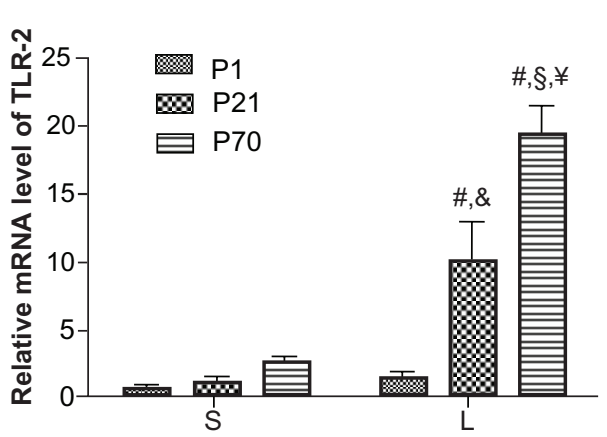

C

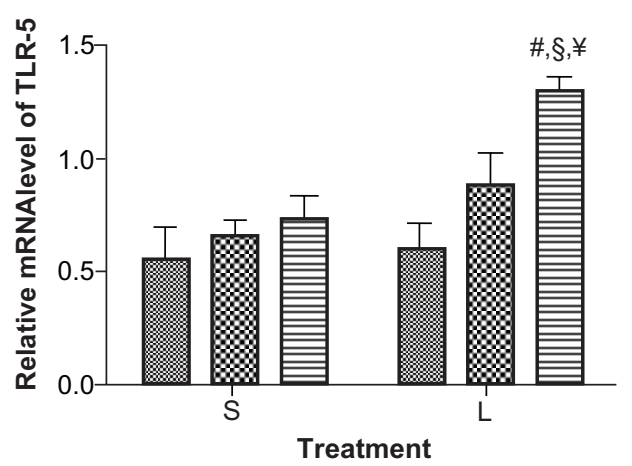

B

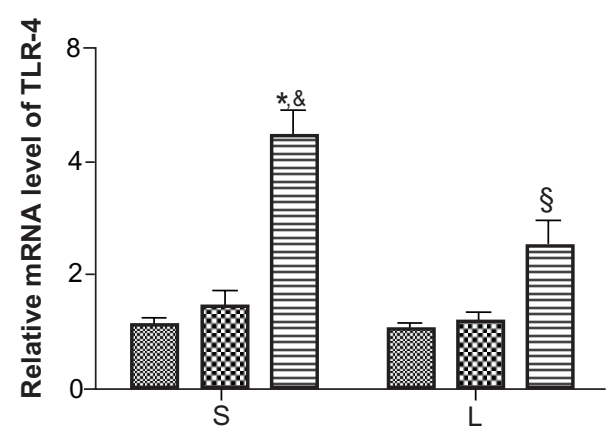

D

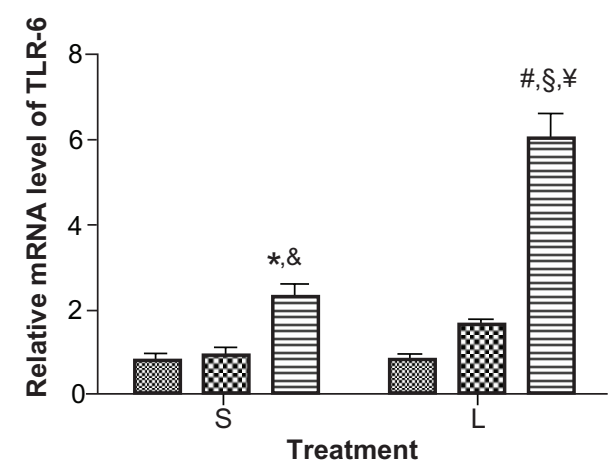

Figure 2 Relative mRNA expression of TLRs in the lung of PI, P2I, and P70 animals. PI, P2I, and P70 animals were treated with saline (S) or 0.25 mg/kg lipopolysaccharide (L) for 2 hours. Relative mRNA levels of TLR-2 (A), TLR-4 (B), TLR-5 (C), and TLR-6 (D) in the lung of these animals were measured by real-time reverse transcription PCR. Notes: Data were presented as means $\pm \mathrm{SE} ; \mathrm{n}=4$. *Represents significant difference from PI $\mathrm{S}$ at $P<0.05$; ${ }^{*}$ represents significant difference from $\mathrm{PI} \mathrm{L} ;{ }^{{ }^{*}}$ represents significant difference from P2I S; ${ }^{*}$ represents significant difference from P2I L; §represents significant difference from P70 S (two-way ANOVA followed by Bonferroni post-test). Abbreviations: mRNA, messenger RNA; PI, postnatal day I; PCR, polymerase chain reaction; TLR, toll-like receptor.

(Figure 3 and Table 1). LPS treatment enhanced the mRNA expression of CD14, MyD88, and LBP in P70 lung by 2.75-, 1.48-, and 1.74-fold, respectively. LPS treatment also enhanced the mRNA expression of CD14 and MyD88 in P21 lung by 2.46- and 1.31-fold, respectively. In contrast, LPS treatment did not significantly affect the mRNA expression of CD14, MyD88, and LBP in P1 lung at 2 hours postinjection (Figure 3 and Table 1).

\section{Expression of pro-inflammatory cytokines in the lung of postnatal animals}

We then examined the mRNA expression of pro-inflammatory cytokines, namely TNF- $\alpha$, IL-1 $\beta$, and IL-6, in the lung of P1, P21, and P70 animals using semiquantitative reverse transcription PCR. Baseline expression of TNF- $\alpha$ and IL-6 was hardly detectable in P1 lung and remained low in P21 and P70 lung (Figure 4B and D). While the baseline level of IL-1 $\beta$ was also low in P1 lung, it was elevated by 4.38- and 6.31-fold in the P21 and P70 lung, respectively (Figure 4C and Table 1). LPS treatment significantly enhanced the mRNA expression of TNF- $\alpha$ by 19.06-, 10.21-, and 10.75-fold, and upregulated the mRNA expression of IL-1 $\beta$ by 9.54-, 4.28-, and 3.17-fold in P1, P21, and P70 lungs, respectively, compared with their saline controls. LPS treatment also enhanced the mRNA expression of IL- 6 by 21.71- and 11.15-fold in P21 and P70 lungs, respectively, without significant effects on P1 lung. Additionally, the mRNA expression of IL-1 $\beta$ and IL-6 in LPS-treated P1 lung was 1.97- and 43.09-fold lower than that in LPS-treated P21 lung, respectively, and the mRNA expression of TNF- $\alpha$, IL-1 $\beta$, and IL-6 in LPS-treated P1 lung was 2.19-, 2.10-, and 45.56-fold lower than that in LPS-treated P70 lung, respectively, at 2 hours postinjection (Figure 4 and Table 1).

\section{Expression of anti-inflammatory cytokines in the lung of postnatal animals}

We then analyzed the transcriptional response of anti-inflammatory cytokines, namely IL-10 and IL-1ra, in the lung of P1, P21, and P70 animals using semiquantitative reverse transcription PCR. Both IL-10 and IL-1ra were found to be constitutively expressed in the lung of animals at different ages and the baseline mRNA expression of IL-10 in P1 lung was 3.98-fold lower than that in P70 lung (Figure 5B and Table 1). LPS treatment significantly enhanced the mRNA level of 
Table I Fold changes in the relative mRNA levels of inflammatory genes in the lung of animals at PI, P2I, and P70 following treatment with saline (S) or lipopolysaccharide (L)

\begin{tabular}{|c|c|c|c|c|c|c|c|}
\hline Gene name & PI L vs PI S & P2I L vs P2I S & P70 L vs P70 S & P2I S vs PI S & P70 S vs PI S & P2I L vs PI L & P70 L vs PI L \\
\hline$T L R-2^{a}$ & 2.00 & $6.18^{*}$ & $8.59 *$ & 1.87 & 3.91 & $5.80 *$ & $16.82 *$ \\
\hline$T L R-4^{a}$ & -1.09 & -1.20 & $-1.75^{*}$ & 1.27 & $3.93 *$ & 1.14 & $2.44^{*}$ \\
\hline$T L R-5^{a}$ & 1.18 & 1.37 & $1.8 I^{*}$ & 1.26 & $\mathrm{I} .47$ & 1.47 & $2.26 *$ \\
\hline$T L R-6^{a}$ & 1.01 & 1.80 & $2.56^{*}$ & 1.10 & $2.7 I^{*}$ & 1.97 & $6.88^{*}$ \\
\hline$\angle B P^{a}$ & 1.16 & -1.06 & $\mathrm{I} .74^{*}$ & $5.19 *$ & $5.99 *$ & $4.21^{*}$ & $8.99 *$ \\
\hline$M y D 88^{b}$ & -1.06 & $1.31 *$ & I.48* & 1.09 & 1.08 & $1.52 *$ & I.70* \\
\hline$C D / 4^{b}$ & 1.19 & $2.46 *$ & $2.75^{*}$ & 1.04 & 1.12 & $2.13^{*}$ & $2.58^{*}$ \\
\hline$T N F-\alpha^{b}$ & $19.06 *$ & $10.21 *$ & $10.75^{*}$ & 2.64 & 3.89 & $\mathrm{I} .4 \mathrm{I}$ & $2.19 *$ \\
\hline$I L-I \beta^{b}$ & $9.54^{*}$ & $4.28^{*}$ & $3.17^{*}$ & $4.38^{*}$ & $6.31 *$ & $1.97^{*}$ & $2.10 *$ \\
\hline$I L-6^{b}$ & 1.17 & $21.71 *$ & $11.15^{*}$ & 2.32 & 4.77 & $43.09 *$ & $45.56^{*}$ \\
\hline$I L-10^{b}$ & $3.76 *$ & $5.85^{*}$ & $2.35^{*}$ & 1.78 & $3.98 *$ & $2.77^{*}$ & $2.49 *$ \\
\hline IL-I $r a^{b}$ & 1.73 & $2.30 *$ & $2.57^{*}$ & 2.31 & 2.82 & $3.07 *$ & $4.18^{*}$ \\
\hline$M I P-I \beta^{b}$ & 2.45 & 8.98* & $3.44 *$ & 1.99 & $4.49 *$ & $7.31 *$ & $6.32^{*}$ \\
\hline$M C P I^{b}$ & -1.01 & $4.94 *$ & $2.78^{*}$ & $-2.63^{*}$ & $-1.79 *$ & I.88* & $1.57^{*}$ \\
\hline$M I P-2^{b}$ & $11.79 *$ & $31.89 *$ & $11.04 *$ & -1.08 & 2.48 & $2.52 *$ & $2.33^{*}$ \\
\hline
\end{tabular}

Notes: PI, P2I, and P70 animals were treated with saline $(\mathrm{S})$ or $0.25 \mathrm{mg} / \mathrm{kg}$ lipopolysaccharide (L) for 2 hours. Relative mRNA levels of inflammatory mediators in the lung of these animals were measured by real-time reverse transcription PCR ( $\left.{ }^{a}\right)$ or semi-quantitative reverse transcription PCR (b). Data were presented as fold changes in relative mRNA levels of inflammatory genes between different treatment groups. *Represents significant difference between the treatment groups (two-way ANOVA followed by Bonferroni post-test).

Abbreviations: IL, interleukin; MCP-I, monocyte chemotactic protein-I; MIP, macrophage inflammatory protein; mRNA, messenger RNA; PI, postnatal day I; PCR, polymerase chain reaction; TLR, toll-like receptor; TNF- $\alpha$, tumor necrosis factor- $\alpha$.

IL-10 in the lung of P1, P21, and P70 animals by 3.76-, 5.85-, and 2.35-fold, respectively, and augmented the mRNA level of IL-1ra in the lung of P21 and P70 animals by 2.30- and 2.57-fold, respectively, as compared with their saline controls. The mRNA expression of IL-10 in LPS-treated P1 lung was 2.77- and 2.49-fold lower than that in LPS-treated P21 and P70 lung, respectively, and the mRNA expression of IL-1 ra in LPS-treated P1 lung was 3.07- and 4.18-fold lower than that in LPS-treated P21 and P70 lung, respectively, at 2 hours postinjection (Figure 5 and Table 1).

\section{Ratio of proinflammatory to anti-inflammatory cytokines in the lung of postnatal animals}

We also calculated the ratios of relative mRNA levels of proinflammatory to anti-inflammatory cytokines in the lung of P1, P21, and P70 animals following treatment with saline or LPS. The ratios of TNF- $\alpha: I L-10$, TNF- $\alpha: I L-1$ ra,

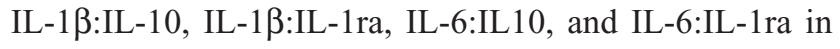
the lung of P1, P21, and P70 animals treated with saline were not significantly different (Table 2). However, the ratio of IL-6:IL-10 in P21 (1.14) and P70 lung (1.28) was significantly higher than that in P1 lung (0.07) at 2 hours following LPS treatment. The ratio of IL-6:IL-1ra in P21 (0.99) and P70 lung (0.71) was also significantly higher than that in P1 lung (0.06) at 2 hours following LPS treatment. However, the ratio of TNF- $\alpha$ :IL-10 in P1 lung (1.46) was slightly higher than that in P21 (0.75) and P70 lung (1.20) and the ratios of TNF- $\alpha: I L-1$ ra, IL-1 $\beta$ :IL-10, and IL-1 $\beta$ :IL-1 ra were not significantly different in P1, P21, and P70 lung at 2 hours following LPS treatment (Table 2).

\section{Expression of chemokines in the lung of postnatal animals}

We then analyzed the relative mRNA levels of chemokines, namely MIP-1 $\beta$, MIP-2, and MCP-1, in the lung of P1, $\mathrm{P} 21$, and $\mathrm{P} 70$ animals using semi-quantitative reverse transcription PCR. The baseline mRNA expression of MIP-2 was barely detectable in the lung of animals at the different ages examined (Figure 6C). The baseline mRNA expression of MCP-1 in P1 lung was 2.63- and 1.79-fold higher than that in P21 and P70 lung, respectively, while the baseline mRNA level of MIP-1 $\beta$ in P1 lung was 4.49-fold lower than that in P70 lung (Figure 6B and D and Table 1). LPS treatment significantly enhanced the mRNA expression of MIP-1 $\beta$ by 8.98- and 3.44-fold, and increased the mRNA expression of MCP-1 by 4.94- and 2.78-fold, respectively, in P21 and P70 lung while the mRNA levels of MIP-1 $\beta$ and MCP-1 in P1 lung were not significantly affected at 2 hours following LPS treatment. LPS treatment also upregulated the mRNA level of MIP-2 in P1, P21, and P70 lung by 11.79-, 31.89-, and 11.04-fold, respectively. The mRNA expression of MIP-1 $\beta$, MIP-2, and MCP-1 in LPS-treated P21 lung was 
A

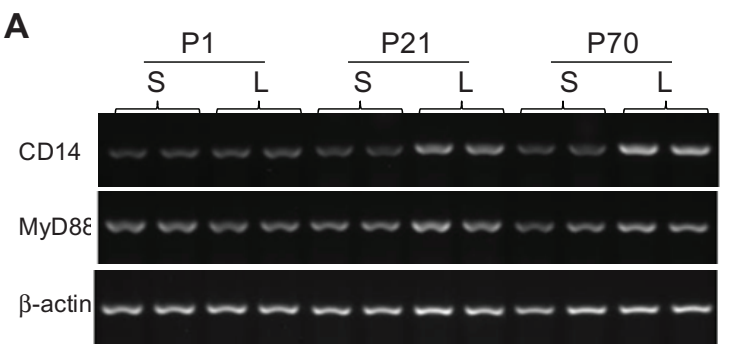

\section{C}

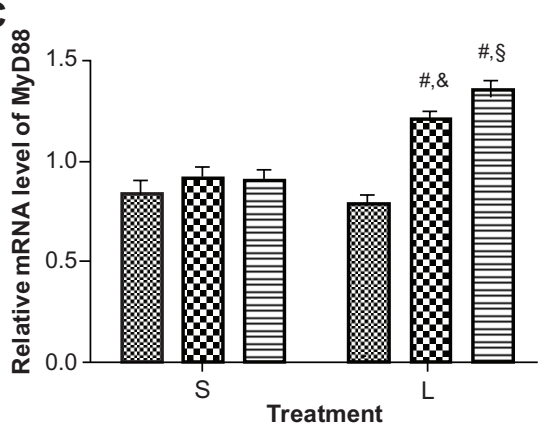

B

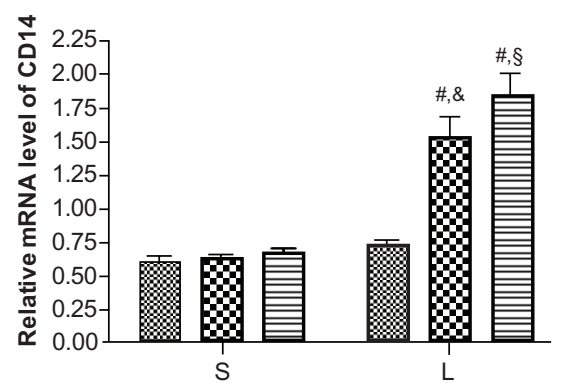

D

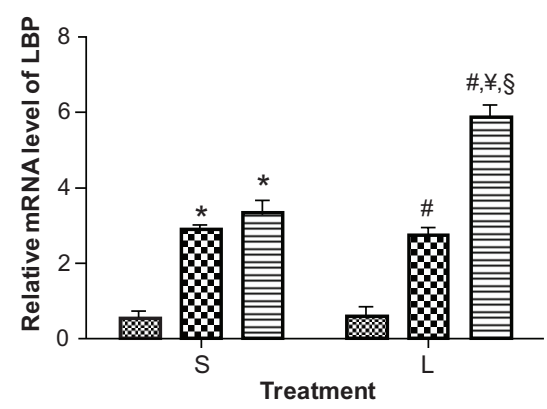

Figure 3 Relative mRNA expression of CDI4, MyD88, and LBP in the lung of PI, P2I, and P70 animals. PI, P2I, and P70 animals were treated with saline (S) or 0.25 mg/kg lipopolysaccharide (L) for 2 hours. Relative mRNA levels of CDI4 (A and B) and MyD88 (A and $\mathbf{C})$ in the lung of these animals were measured by semi-quantitative reverse transcription PCR and the relative mRNA level of LBP (D) was measured by real-time reverse transcription PCR.

Notes: Data were presented as means $\pm \mathrm{SE} ; \mathrm{n}=4$. *Represents significant difference from PI $\mathrm{S}$ at $P<0.05$; ${ }^{*}$ represents significant difference from PI L; ${ }^{\circledR}$ represents significant difference from P2I S; ${ }^{*}$ represents significant difference from P2I L; ${ }^{\S}$ represents significant difference from P70 S (two-way ANOVA followed by Bonferroni post-test).

Abbreviations: LBP, lipopolysaccharide binding protein; MyD88, myeloid differentiation marker 88; PI, postnatal day I; mRNA, messenger RNA; PCR, polymerase chain reaction.

A

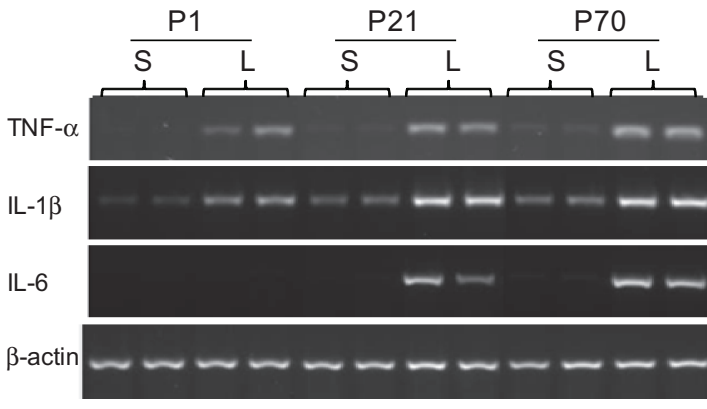

C

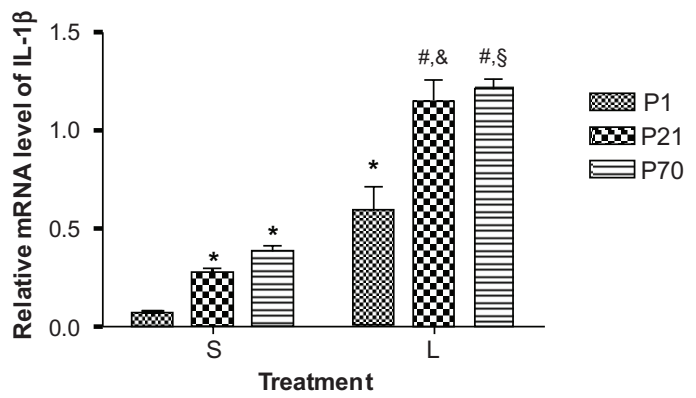

B

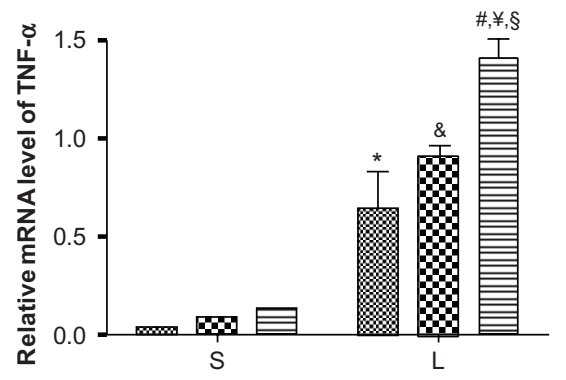

D

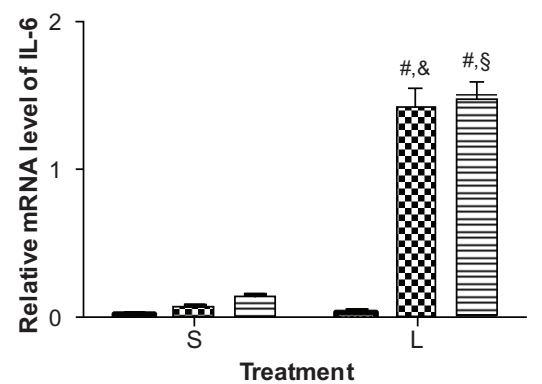

Figure 4 Relative mRNA expression of proinflammatory cytokines in the lung of PI, P2I, and P70 animals. PI, P2I, and P70 animals were treated with saline (S) or 0.25 mg/kg lipopolysaccharide (L) for 2 hours. Relative mRNA levels of TNF- $\alpha$ (A and B), IL-I $\beta$ (A and $\mathbf{C})$, and IL-6 (A and $\mathbf{D})$ in the lung of these animals were measured by semiquantitative reverse transcription PCR.

Notes: Data were presented as means $\pm \mathrm{SE} ; \mathrm{n}=4$. *Represents significant difference from PI S at $P<0.05$; ${ }^{*}$ represents significant difference from PI L; ${ }^{\&}$ represents significant difference from P2I S; ${ }^{*}$ represents significant difference from P2I L; ${ }^{\S}$ represents significant difference from P70 S (two-way ANOVA followed by Bonferroni post-test).

Abbreviations: IL, interleukin; PI, postnatal day I; PCR, polymerase chain reaction; TNF, tumor necrosis factor. 
A

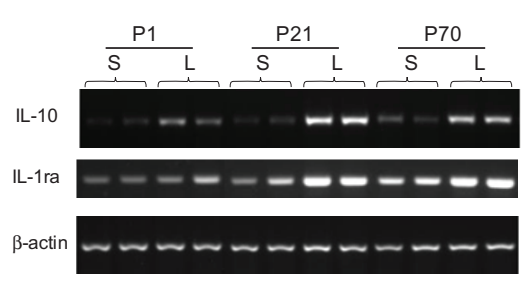

B

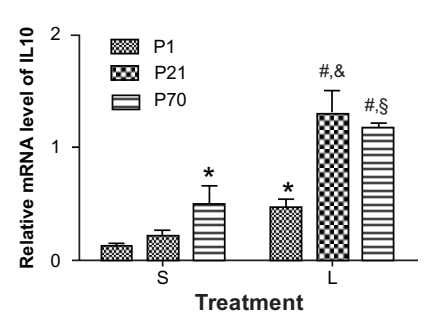

C

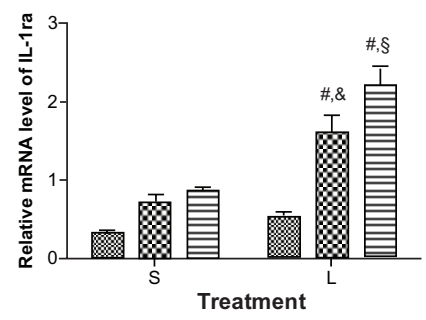

Figure 5 Relative mRNA expression of anti-inflammatory cytokines in the lung of PI, P2I, and P70 animals. PI, P2I, and P70 animals were treated with saline (S) or $0.25 \mathrm{mg} / \mathrm{kg}$ lipopolysaccharide (L) for 2 hours. Relative mRNA levels of IL-I0 (A and B) and IL-I ra (A and $\mathbf{C})$ in the lung of these animals were measured by semiquantitative reverse transcription PCR.

Notes: Data were presented as means \pm SE; $n=4$. *Represents significant difference from PI S at $P<0.05$; ${ }^{*}$ represents significant difference from PI L; ${ }^{\circledR}$ represents significant difference from P2I S; ${ }^{\circledR}$ represents significant difference from P70 S (two-way ANOVA followed by Bonferroni post-test).

Abbreviations: IL-I0, interleukin-I0; IL-I ra, interleukin-I receptor antagonist; mRNA, messenger RNA; PI, postnatal day I.

higher than that in LPS-treated P1 lung by 7.31-, 2.52-, and 1.88 -fold, respectively, and the mRNA expression of MIP-1 $\beta$, MIP-2, and MCP-1 in LPS-treated P70 lung was higher than that in LPS-treated P1 lung by 6.32-, 2.33-, and 1.57-fold, respectively (Figure 6 and Table 1).

\section{Enzymatic activity of myeloperoxidase in the lung of postnatal animals}

Myeloperoxidase (MPO) is a peroxidase enzyme most abundantly expressed in neutrophils. In order to assess the infiltration of neutrophils into the lung, we extracted proteins from the lung tissues and performed MPO enzymatic activity assay. The basal MPO activity was comparable in the lung of P1 (3.19 $\pm 0.34 \mathrm{U} / \mathrm{mg})$, P2 $1(3.64 \pm 0.63 \mathrm{U} / \mathrm{mg})$, and P70 $(3.09 \pm 1.18 \mathrm{U} / \mathrm{mg})$ (Figure 7). LPS treatment significantly increased the MPO activity in P70 lung $(15.9 \pm 2.42 \mathrm{U} / \mathrm{mg})$ as compared to its saline controls. LPS treatment also appeared to increase the MPO activity in P1 $(4.64 \pm 1.43 \mathrm{U} / \mathrm{mg})$ and P21 lung $(5.62 \pm 1.34 \mathrm{U} / \mathrm{mg})$, yet the increase was not statistically significant. Additionally, MPO activity in LPS-treated P70 lung was significantly higher than LPS-treated P1 and P21 lungs (Figure 7).

\section{Discussion}

Bacterial sepsis is a significant cause of neonatal morbidity and mortality, which is in large part secondary to developmentally immature host defense mechanisms. ${ }^{21,22}$ The main objective of the present study was to examine the postnatal development of TLR-4-mediated innate immunity in the lung. Our study showed that LPS-induced transcriptional response of pro-inflammatory cytokines, namely IL-1 $\beta$, IL-6, and TNF- $\alpha$, and anti-inflammatory cytokines, namely IL-10 and IL-1ra, in P1 lung, was much reduced compared to that in P21 and P70 lung. Consistently, LPS-induced cytokine response in cord blood has a different profile from the adult venous blood. ${ }^{23}$ Cord blood monocytes have been shown to be less responsive to LPS by producing diminished levels of cytokines than adult human peripheral blood mononuclear cells (hPBMCs). ${ }^{9,24}$ Neonatal macrophages have also been reported to produce less IL- $1 \beta$ and TNF- $\alpha$ than adult macrophages from mice. ${ }^{25}$ Furthermore, our study found that while the ratio of relative mRNA levels of TNF- $\alpha$ :IL-10 in P1 lung was slightly higher than that in P21 or P70 lung following LPS stimulation, the ratio of IL-6:IL10 and IL-6:IL-1ra in P1 lung was significantly lower than that in P21 and P70 lung at 2

Table 2 Ratios of the relative mRNA levels of proinflammatory to anti-inflammatory cytokines in the lung of PI, P2I, and P70 animals following treatment with saline $(S)$ or lipopolysaccharide $(L)$

\begin{tabular}{|c|c|c|c|c|c|c|}
\hline Ratio & PIS & P2I S & P70 S & PI L & P2I L & P70 L \\
\hline TNF- $\alpha: I L-10$ & $0.27 \pm 0.04$ & $0.43 \pm 0.09$ & $0.32 \pm 0.07$ & $1.46 \pm 0.48$ & $0.75 \pm 0.1 I^{*}$ & $1.20 \pm 0.06 *$ \\
\hline TNF- $\alpha: I L-I$ ra & $0.12 \pm 0.03$ & $0.13 \pm 0.01$ & $0.15 \pm 0.01$ & $1.18 \pm 0.28$ & $0.62 \pm 0.12$ & $0.66 \pm 0.05$ \\
\hline IL-I $\beta: I L-10$ & $0.50 \pm 0.06$ & $1.30 \pm 0.21$ & $0.97 \pm 0.23$ & $1.35 \pm 0.36$ & $0.93 \pm 0.13$ & $1.05 \pm 0.04$ \\
\hline IL-I $\beta: I L-I$ ra & $0.21 \pm 0.02$ & $0.40 \pm 0.03$ & $0.46 \pm 0.03$ & $1.15 \pm 0.28$ & $0.79 \pm 0.20$ & $0.60 \pm 0.09$ \\
\hline IL-6:IL-I0 & $0.25 \pm 0.05$ & $0.30 \pm 0.02$ & $0.33 \pm 0.07$ & $0.07 \pm 0.03$ & $1.14 \pm 0.10^{*}$ & $1.28 \pm 0.04 *$ \\
\hline IL-6:IL-I ra & $0.10 \pm 0.02$ & $0.10 \pm 0.01$ & $0.16 \pm 0.01$ & $0.06 \pm 0.02$ & $0.99 \pm 0.25 *$ & $0.71 \pm 0.06^{*}$ \\
\hline
\end{tabular}

Notes: PI, P2 I, and P70 animals were treated with saline (S) or $0.25 \mathrm{mg} / \mathrm{kg}$ lipopolysaccharide (L) for 2 hours. Relative mRNA levels of proinflammatory and anti-inflammatory cytokines in the lung of these animals were measured by semi-quantitative RT-PCR, and the ratios of relative mRNA levels of proinflammatory to anti-inflammatory cytokines were calculated. Data were presented as means \pm SE. *Represents significant difference from PI L (two-way ANOVA followed by Bonferroni post-test).

Abbreviations: IL, interleukin; mRNA, messenger RNA; PI, postnatal day I; PCR, polymerase chain reaction; TNF- $\alpha$, tumor necrosis factor- $\alpha$. 
A

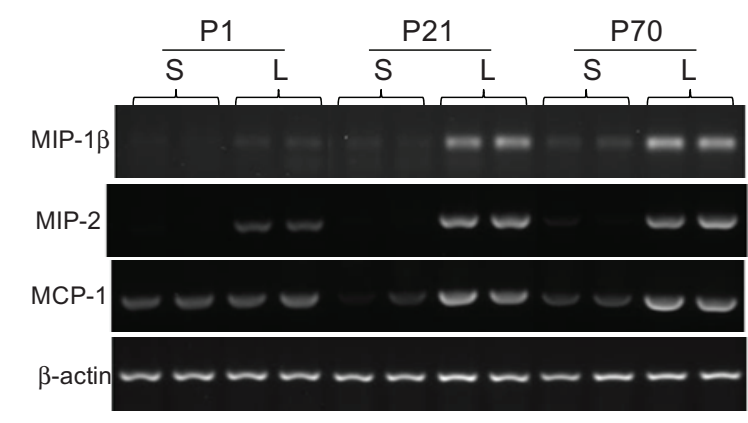

C

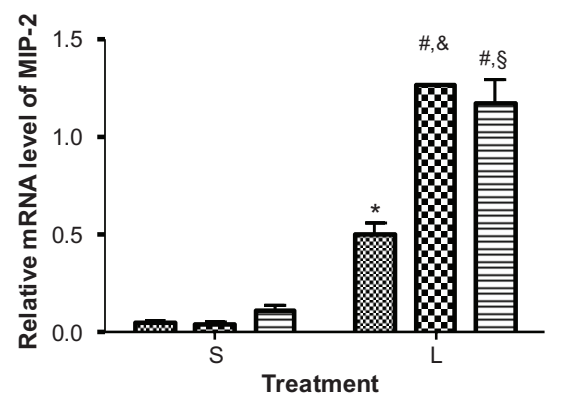

B

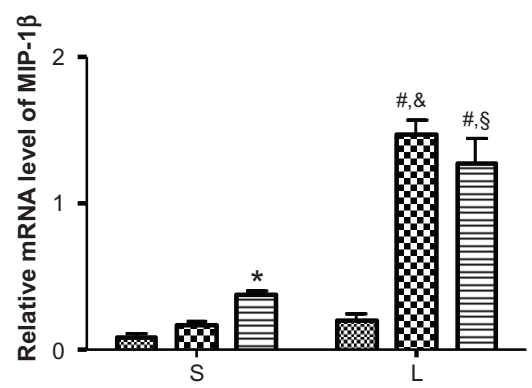

D

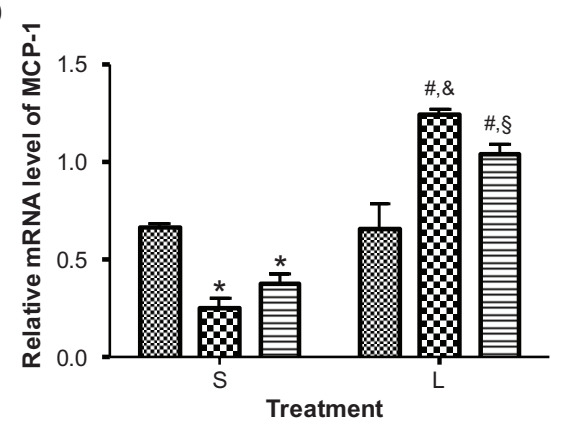

Figure 6 Relative mRNA expression of chemokines in the lung of PI, P2I, and P70 animals. PI, P2I, and P70 animals were treated with saline (S) or 0.25 mg/kg lipopolysaccharide (L) for 2 hours. Relative mRNA levels of MIP-I $\beta$ (A and $\mathbf{B})$, MIP-2 (A and $\mathbf{C})$, and MCP-I (A and $\mathbf{D})$ in the lung of these animals were measured by semiquantitative reverse transcription PCR.

Notes: Data were presented as means $\pm \mathrm{SE} ; \mathrm{n}=4$. *Represents significant difference from PI $\mathrm{S}$ at $P<0.05$; ${ }^{*}$ represents significant difference from PI L; ${ }^{\circledR}$ represents significant difference from P2I S; §represents significant difference from P70 S (two-way ANOVA followed by Bonferroni post-test).

Abbreviations: MCP-I, monocyte chemotactic protein-I; MIP, macrophage inflammatory protein; PI, postnatal day I.

hours following LPS stimulation, which may contribute to their increased susceptibility to neonatal infections. Consistently, the IL-6:IL-10 ratio has been reported to be significantly higher in septic patients with multiple organ failure in comparison with patients without multiple organ failure. ${ }^{26}$

Our study also showed that LPS-induced transcriptional response of chemokines, namely MIP-1 1 , MIP-2, and MCP-
1, in P1 lung was much reduced compared to that in P21 and P70 lung. MPO activity in LPS-treated P1 lung was also significantly attenuated compared to that in LPS-treated P70 lung, suggesting that neutrophil recruitment into neonatal lung was impaired as compared to that in older animals.

In parallel, our study showed that the basal and LPSinduced mRNA level of LBP in P1 lung was significantly

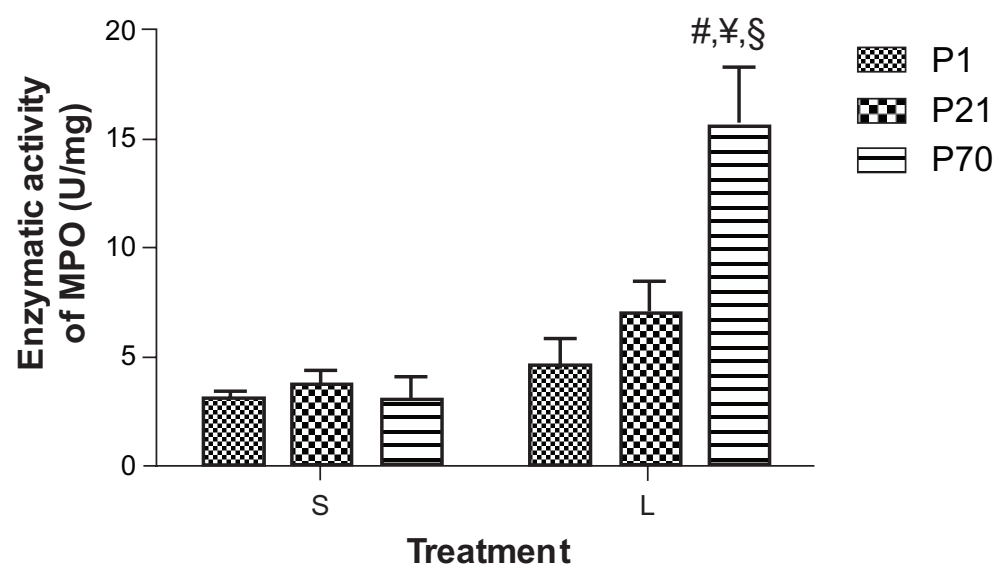

Figure 7 Enzymatic activity of myeloperoxidase (MPO) in the lung of PI, P2I, and P70 animals. PI, P2I, and P70 animals were treated with saline (S) or 0.25 mg/kg lipopolysaccharide (L) for 2 hours. MPO activity in the lung of these animals was then measured by enzymatic assay.

Notes: Data were presented as means \pm SE; $n=4$. "Represents significant difference from PI L; ${ }^{\sharp}$ represents significant difference from P2I L; ${ }^{\S}$ represents significant difference from P70 S (two-way ANOVA followed by Bonferroni post-test).

Abbreviation: PI, postnatal day I. 
lower than that in P21 and P70 lung. LBP knockout mice have been reported to experience increased mortality from Gram-negative pneumonia compared with their wild-type controls, and this mortality difference is abolished with systemic LBP gene therapy. ${ }^{27}$ Total lung myeloperoxidase activity is also diminished significantly in LBP knockout mice. ${ }^{28}$ Therefore, a low level of LBP expression in P1 lung may contribute to its reduced induction of cytokines and chemokines following LPS stimulation as compared to older animals.

It is of interest to note that treatment with LPS, a PAMP for TLR-4, augmented the mRNA expression of TLR-2, TLR-5, and TLR-6 in P70 lung at 2 hours postinjection, suggesting that expression of different TLRs in the lung may be cross-regulated. Consistently, stimulation of TLR-5 by bacterial flagellin has been reported to upregulate TLR-2 and TLR-4 mRNA and result in sensitization of mouse intestinal epithelial cell line mICcl2 for subsequent TLR-2 and TLR-4 stimulation. ${ }^{29}$ LPS treatment has also been reported to increase TLR-2 but decrease TLR-4 mRNA in rat liver. ${ }^{30}$ While Matsumura et al reported that LPS stimulation increases TLR-4 expression in murine lung, ${ }^{31}$ Bozinovski et al reported a decline of TLR- 4 mRNA at 2 hours following LPS stimulation and an increase at 4 hours and 24 hours post-LPS injection. ${ }^{32}$ Consistently, TLR-4 mRNA expression was found to be attenuated in P70 lung at 2 hours post-LPS injection in our studies. It is also important to point out that in our study the levels of inflammatory genes were measured at the mRNA level, and that these measurements may not reflect their final protein levels.

\section{Conclusion}

In summary, our study showed that LPS-induced transcriptional response of inflammatory mediators as well as the ratios of relative mRNA levels of IL6:IL-10 and IL6:IL-1ra in the lung of P1 animals were much reduced in magnitude as compared to those in older animals, at 2 hours following LPS treatment, suggesting that LPS-induced inflammatory response in P1 lung was significantly attenuated. In parallel, mRNA expression of LBP in P1 lung was much lower than that in older animals, suggesting that a lower level of LBP may contribute to the attenuated inflammatory response in P1 lung following LPS stimulation as compared to older animals. These studies help to understand the molecular mechanisms underlying the increased susceptibility of neonates to bacterial infections, and may contribute to designing new therapeutic approaches to control infections in neonates.

\section{Acknowledgments}

This work was supported in part by the National Institute of Health Grant R15-HD065643 to HZ.

\section{Disclosure}

The authors report no conflicts of interest in this work.

\section{References}

1. Takeda K, Akira S. TLR signaling pathways. Semin Immunol. 2004; 16(1):3-9.

2. Fitzgerald KA, Palsson-McDermott EM, Bowie AG, et al. Mal (MyD88adapter-like) is required for Toll-like receptor-4 signal transduction. Nature. 2001;413(6851):78-83.

3. Zeytun A, van Velkinburgh JC, Pardington PE, Cary RR, Gupta G. Pathogen-specific innate immune response. Adv Exp Med Biol. 2007; 598:342-357.

4. Arend WP. Interleukin-1 receptor antagonist. Adv Immunol. 1993; 54:167-227.

5. Ng PC, Li K, Wong RP, et al. Proinflammatory and anti-inflammatory cytokine responses in preterm infants with systemic infections. Arch Dis Child Fetal Neonatal. 2003;88(3):F209-F213.

6. Afroza S. Neonatal sepsis - a global problem: an overview. Mymensingh Med J. 2006;15(1):108-114.

7. Lukacs SL, Schoendorf KC, Schuchat A. Trends in sepsis-related neonatal mortality in the United States, 1985-1998. Pediatr Infect Dis J. 2004;23(7):599-603.

8. Yan SR, Qing G, Byers DM, Stadnyk AW, Al-Hertani W, Bortolussi R. Role of MyD88 in diminished tumor necrosis factor alpha production by newborn mononuclear cells in response to lipopolysaccharide. Infect Immun. 2004;72(3):1223-1229.

9. Hebra A, Strange P, Egbert JM, Ali M, Mullinax A, Buchanan E. Intracellular cytokine production by fetal and adult monocytes. J Pediatr Surg. 2001;36(9):1321-1326.

10. Levy O, Zarember KA, Roy RM, Cywes C, Godowski PJ, Wessels MR. Selective impairment of TLR-mediated innate immunity in human newborns: neonatal blood plasma reduces monocyte TNF-alpha induction by bacterial lipopeptides, lipopolysaccharide, and imiquimod, but preserves the response to R-848. J Immunol. 2004;173(7):4627-4634.

11. Crespo I, Paiva A, Couceiro A, Pimentel P, Orfao A, Regateiro F. Immunophenotypic and functional characterization of cord blood dendritic cells. Stem Cells Dev. 2004;13(1):63-70.

12. Harju K, Ojaniemi M, Rounioja S, et al. Expression of toll-like receptor 4 and endotoxin responsiveness in mice during perinatal period. Pediatr Res. 2005;57(5 Pt 1):644-648.

13. Lee PT, Holt PG, McWilliam AS. Role of alveolar macrophages in innate immunity in neonates: evidence for selective lipopolysaccharide binding protein production by rat neonatal alveolar macrophages. $\mathrm{Am}$ J Respir Cell Mol Biol. 2000;23(5):652-661.

14. Martin TR, Ruzinski JT, Wilson CB, Skerrett SJ. Effects of endotoxin in the lungs of neonatal rats: age-dependent impairment of the inflammatory response. J Infect Dis. 1995;171(1):134-144.

15. Lee PT, Holt PG, McWilliam AS. Ontogeny of rat pulmonary alveolar macrophage function: evidence for a selective deficiency in il-10 and nitric oxide production by newborn alveolar macrophages. Cytokine. 2001;15(1):53-57.

16. Le Rouzic V, Corona J, Zhou H. Postnatal development of hepatic innate immune response. Inflammation. 2011;34(6):576-584.

17. Surriga O, Ortega A, Jadeja V, Bellafronte A, Lasala N, Zhou H. Altered hepatic inflammatory response in the offspring following prenatal LPS exposure. Immunol Lett. 2009;123(1):88-95.

18. Lasala N, Zhou H. Effects of maternal exposure to LPS on the inflammatory response in the offspring. J Neuroimmunol. 2007; 189(1-2):95-101. 
19. Ambrosini A, Louin G, Croci N, Plotkine M, Jafarian-Tehrani M. Characterization of a rat model to study acute neuroinflammation on histopathological, biochemical and functional outcomes. J Neurosci Methods. 2005;144(2):183-191.

20. Sheridan BC, McIntyre RC Jr, Meldrum DR, Fullerton DA. L-arginine prevents lung neutrophil accumulation and preserves pulmonary endothelial function after endotoxin. Am J Physiol. 1998;274(3 Pt 1): L337-L342.

21. Chirico G, Cortinovis S, Fonte C, Giudici G. Bacterial sepsis. J Chemother. 2007;19 Suppl 2:28-30.

22. Bogaert D, Weinberger D, Thompson C, Lipsitch M, Malley R. Impaired innate and adaptive immunity to Streptococcus pneumoniae and its effect on colonization in an infant mouse model. Infect Immun. 2009; 77(4):1613-1622.

23. Belderbos ME, van Bleek GM, Levy O, et al. Skewed pattern of Tolllike receptor 4-mediated cytokine production in human neonatal blood: low LPS-induced IL-12p70 and high IL-10 persist throughout the first month of life. Clin Immunol. 2009;133(2):228-237.

24. Sadeghi K, Berger A, Langgartner M, et al. Immaturity of infection control in preterm and term newborns is associated with impaired toll-like receptor signaling. J Infect Dis. 2007;195(2):296-302.

25. Chelvarajan RL, Collins SM, Doubinskaia IE, et al. Defective macrophage function in neonates and its impact on unresponsiveness of neonates to polysaccharide antigens. J Leukoc Biol. 2004;75(6) 982-994.
26. Loisa P, Rinne T, Laine S, Hurme M, Kaukinen S. Anti-inflammatory cytokine response and the development of multiple organ failure in severe sepsis. Acta Anaesthesio Scand. 2003;47(3):319-325.

27. Hemmila MR, Kim J, Sun JM, et al. Gene therapy with lipopolysaccharide binding protein for gram-negative pneumonia: respiratory physiology. J Trauma. 2006;61(3):598-605; discussion 6.

28. Fan MH, Klein RD, Steinstraesser L, et al. An essential role for lipopolysaccharide-binding protein in pulmonary innate immune responses. Shock. 2002;18(3):248-254.

29. van Aubel RA, Keestra AM, Krooshoop DJ, van Eden W, van Putten JP. Ligand-induced differential cross-regulation of Toll-like receptors 2, 4 and 5 in intestinal epithelial cells. Mol Immunol. 2007;44(15): 3702-3714.

30. Peng JH, Hu YY, Cheng Y, et al. Effect of JIANPI HUOXUE decoction on inflammatory cytokine secretion pathway in rat liver with lipopolysaccharide challenge. World J Gastroenterol. 2008;14(12): 1851-1857.

31. Matsumura T, Ito A, Takii T, Hayashi H, Onozaki K. Endotoxin and cytokine regulation of toll-like receptor (TLR) 2 and TLR4 gene expression in murine liver and hepatocytes. J Interferon Cytokine Res. 2000;20(10):915-921.

32. Bozinovski S, Jones J, Beavitt SJ, Cook AD, Hamilton JA, Anderson GP. Innate immune responses to LPS in mouse lung are suppressed and reversed by neutralization of GM-CSF via repression of TLR-4. Am J Physiol Lung Cell Mol Physiol. 2004;286(4):L877-L885.
Journal of Inflammation Research

\section{Publish your work in this journal}

The Journal of Inflammation Research is an international, peer-reviewed open-access journal that welcomes laboratory and clinical findings on the molecular basis, cell biology and pharmacology of inflammation including original research, reviews, symposium reports, hypothesis formation and commentaries on: acute/chronic inflammation; mediators of inflamma-

\section{Dovepress}

tion; cellular processes; molecular mechanisms; pharmacology and novel anti-inflammatory drugs; clinical conditions involving inflammation. The manuscript management system is completely online and includes a very quick and fair peer-review system. Visit http://www.dovepress.com/ testimonials.php to read real quotes from published authors. 